\title{
Is the question of phenobarbital as potential liver cancer risk factor for humans really resolved?
}

\author{
Albert Braeuning ${ }^{1,2} \cdot$ Michael Schwarz ${ }^{2}$
}

Received: 23 March 2016 / Accepted: 13 April 2016 / Published online: 22 April 2016

(C) Springer-Verlag Berlin Heidelberg 2016

Human relevance of tumor promotion in rodent bioassays is sometimes controversially discussed as, for example, in the case of the antiepileptic drug phenobarbital (PB), which causes liver tumors in mice following chronic administration. A new study by Braeuning et al. recently published in Archives of Toxicology now provides further mechanistic insight into the complexity of PB-mediated rodent hepatocarcinogenesis by demonstrating that hepatocellular adenoma (HCA) is promoted by chronic PB treatment of mice with a liver-specific deficiency of APC, a negative regulator of the oncogenic Wnt/ $\beta$-catenin signaling pathway, whereas the same treatment inhibited rather than promoted the formation of hepatocellular carcinoma (HCC) in this mouse model (Braeuning et al. 2016).

In fact, "tumor promotion" is a potentially misleading term, which has been historically defined in an operational manner, i.e., as a process during which, under the influence of a "tumor promoter", tumors appear earlier, are more frequent and larger in size. Importantly, this activity of tumor promoters is generally very specific to certain cell types, tissues and organs. At the molecular level, nongenotoxic carcinogens or tumor promoters act via diverse mechanisms, for example through modulation of endogenous signaling pathways including hormonal regulation, inhibition of apoptosis or induction of cell proliferation. A tumor-promoting substance thereby enables initiated cells to gain a selective advantage over the surrounding normal

Albert Braeuning

Albert.Braeuning@bfr.bund.de

1 Department of Food Safety, Federal Institute for Risk Assessment, Max-Dohrn-Str. 8-10, 10589 Berlin, Germany

2 Department of Toxicology, University of Tübingen, Wilhelmstr, 56, 72074 Tübingen, Germany cells. However, this view falls short of the complexity of the phenomenon of tumor promotion, as highlighted by the abovementioned study.

A chemical might generate "environmental" conditions in its target organ, for example the liver, which are beneficial for a certain type of tumor cell, i.e., a cell harboring a particular genetic alteration leading to activation or inactivation of a certain signaling pathway, while at the same time being detrimental for another type of tumor cell bearing a different mutation which leads to a different profile of cellular signaling. This is true not only for the differences between HCA and HCC formation (Braeuning et al. 2016), but even for different molecular subtypes of HCA in mouse liver: Previous work from our group has shown that treatment with PB specifically promotes the outgrowth of HCA with $C$ tnnbl mutations which lead to activated $\beta$-catenin signaling (Aydinlik et al. 2001), whereas single hepatocytes with activated $\beta$-catenin do not have a selective advantage (or even a disadvantage) in the environment of a non-PBexposed liver (Braeuning et al. 2016, 2010; Schreiber et al. 2011). By contrast, HCA with activated mitogen-activated protein kinase signaling, e.g., because of activating mutations in Ha-ras or B-raf, possess a pronounced proliferative advantage in the absence of PB which is turned into opposite upon PB treatment which blocks the outgrowth of the latter tumor subpopulation (Aydinlik et al. 2001). That means that the change in "environmental" conditions to the liver by PB does not promote every type of tumor but rather specifically selects for a certain tumor subpopulation which gains an advantage under the specific conditions of PB treatment. Thus, speaking of "tumor promotion" is not fully appropriate. Instead, the term "tumor selection" would cover the phenomenon more precisely.

This view, however, substantially complicates risk assessment. A "tumor promoter" will be regarded as 
potentially harmful in general, whereas a "tumor selector" might combine carcinogenic and chemopreventive properties, with the molecular characteristics of an individual tumor cell determining whether the substance will exert adverse or beneficial effects.

Human epidemiology for PB was not able to reveal carcinogenic effects of the compound in humans, as discussed, e.g., in Braeuning et al. (2014). This fact is often used as key argument for the assumption that PB is not carcinogenic to humans, a view which is not in full agreement with the conclusions by the International Agency for the Research on Cancer (IARC), which has classified PB as a class 2B carcinogen (IARC 2001). However, the apparent lack of increase in incidence in liver cancer in patients treated for prolonged periods of time with PB as an anticonvulsive drug might, in principle, be explained not only by a lack of effect of the agent in humans, but also by a scenario in which PB acts as a tumor selector which, by promoting one type of tumor while inhibiting another one, results in an overall unchanged tumor incidence. To our opinion, there is no solid evidence to justify the assumption that the phenomenon of tumor selection by cancer risk factors is limited to rodents. Recent evidence has demonstrated, for example, that alcohol-related HCC in humans are significantly enriched in CTNNBI mutations (Schulze et al. 2015).

The mechanisms by which PB causes tumor promotion or inhibition are still not fully understood. Without such deeper mechanistic understanding, however, it will be difficult if not impossible to appropriately stratify the human population into individuals at risk or not at risk from a substance like PB with tumor-selecting properties.

\section{References}

Aydinlik H, Nguyen TD, Moennikes O, Buchmann A, Schwarz M (2001) Selective pressure during tumor promotion by phenobarbital leads to clonal outgrowth of beta-catenin-mutated mouse liver tumors. Oncogene 20:7812-7816

Braeuning A, Singh Y, Rignall B, Buchmann A, Hammad S, Othman A, von Recklinghausen I, Godoy P, Hoehme S, Drasdo D, Hengstler JG, Schwarz M (2010) Phenotype and growth behavior of residual beta-catenin-positive hepatocytes in livers of betacatenin-deficient mice. Histochem Cell Biol 134:469-481

Braeuning A, Gavrilov A, Brown S, Wolf CR, Henderson CJ, Schwarz M (2014) Phenobarbital-mediated tumor promotion in transgenic mice with humanized CAR and PXR. Toxicol Sci 140:259-270

Braeuning A, Gavrilov A, Geissler M, Wenz C, Colnot S, Templin MF, Metzger U, Romer M, Zell A, Schwarz M (2016) Tumor promotion and inhibition by phenobarbital in livers of conditional Apcdeficient mice. Arch Toxicol. doi:10.1007/s00204-016-1667-1

IARC (2001) Phenobarbital and its sodium salt. IARC monographs on the evaluation of carcinogenic risks to humans. IARC Press, Lyon, France, pp 161-288

Schreiber S, Rignall B, Braeuning A, Marx-Stoelting P, Ott T, Buchmann A, Hammad S, Hengstler JG, Schwarz M, Kohle C (2011) Phenotype of single hepatocytes expressing an activated version of beta-catenin in liver of transgenic mice. J Mol Histol 42:393-400

Schulze K, Imbeaud S, Letouze E, Alexandrov LB, Calderaro J, Rebouissou S, Couchy G, Meiller C, Shinde J, Soysouvanh F, Calatayud AL, Pinyol R, Pelletier L, Balabaud C, Laurent A, Blanc JF, Mazzaferro V, Calvo F, Villanueva A, Nault JC, Bioulac-Sage P, Stratton MR, Llovet JM, Zucman-Rossi J (2015) Exome sequencing of hepatocellular carcinomas identifies new mutational signatures and potential therapeutic targets. Nat Genet 47:505-511 